\title{
Contribution à l'étude des composés liposolubles du lait de bufflonne
}

\author{
par \\ F. ADDEO*, Simonne KUZDZAL-SAVOIE**, L. CHIANESE*, \\ A. MALORNI *** et C. SEPE *
}

\section{INTRODUCTION}

Le nombre élevé d'études analytiques consacrées aux lipides du lait de vache a mis en valeur l'extrême complexité de ces lipides.

Plus de trois cents acides gras ont été identifiés dans la phase lipidique [21] et les études relatives aux composés liposolubles (hydrocarbures, alcools...), révèlent sans cesse de nouveaux constituants.

Le lait de bufflonne a fait l'objet d'un nombre réduit d'études et les constituants lipidiques isolés ou identifiés sont, à ce jour, moins nombreux dans le lait de bufflonne que dans le lait de vache.

Le lait de bufflonne contient en moyenne 8,5 p. 100 de matière grasse, soit approximativement le double de la quantité habituellement trouvée dans le lait de vache [2]. Le lait de bufflonne ne contient pas de $\beta$-carotène; la matière grasse est pratiquement incolore. Le contenu en vitamine A est par contre plus élevé dans le lait de bufflonne que dans le lait de vache [14]. Les quantités de phospholipides sont équivalentes dans les deux laits et voisines de 0,03 g p. 100 de lait [32].

Mincione et al. [29] indiquent pour le lait de bufflonne une valeur moyenne de $0,04 \mathrm{~g}$ et des valeurs extrêmes de 0,036 et $0,052 \mathrm{~g}$ pour $100 \mathrm{~g}$ de lait. Cependant le lait de bufflonne contient un peu moins

* Istituto di Industrie Agrarie, Facoltà di Agraria dell'Università di Napoli, 80055 Portici, Italie. - ** Laboratoire de Biochimie et Technologie Laitières, I.N.R.A.-C.N.R.Z., 78350 Jouy-en-Josas, France. - *** Laboratorio del C.N.R. per la Chimica di molecole di interesse biologico, Via Troiano 2, Arco Felice (Napoli), Italie. 
de phosphatidyléthanolamine et de lécithine mais un peu plus de sphingomyéline que le lait de vache [31] et se rapproche donc du lait de femme par sa composition phospholipidique. Le taux de cholestérol est relativement faible. Mincione et al. [27], dans une étude portant sur 21 échantillons de lait de mélange provenant de trois provinces d'Italie, indiquent une valeur moyenne de $102 \mathrm{mg}$ pour $100 \mathrm{~g}$ de matière grasse avec des valeurs extrêmes de 55 et $147 \mathrm{mg}$. Selon ces auteurs, le lait de bufflonne contient également des quantités réduites de campestérol, de stigmastérol, de $\beta$-sitostérol et de $\Delta_{5}$-avénastérol, respectivement 1,$64 ; 0,42 ; 1,45$ et $0,34 \mathrm{p} .100$ des stérols totaux.

Les proportions relatives des acides gras du lait de bufflonne sont voisines de celles du lait de vache. Toutefois la proportion moyenne d'acide oléique est un peu plus faible, et la proportion moyenne d'acide palmitique un peu plus forte que dans le lait de vache $[10,11]$. Pour des proportions équivalentes d'acide butyrique et d'acide caproïque les proportions des acides comptant 8 et 10 atomes de carbone apparaissent légèrement plus faibles dans le lait de bufflonne que dans le lait de vache; le rapport 16:0/8:0 apparaît donc plus élevé dans le lait de bufflonne que dans le lait de vache [3].

Il résulte de ces différentes observations que le point de fusion de la matière grasse du lait de bufflonne est un peu plus élevé que le point de fusion de la matière grasse du lait de vache alors que l'indice d'iode et l'indice de Polenske sont un peu plus faibles dans le lait de bufflonne que dans le lait de vache. Un même schéma de répartition des acides polyinsaturés à longue chaîne est observé lors de l'analyse par chromatographie en phase gazeuse (avec une phase stationnaire de succinate de butanédiol ou BDS) des esters méthyliques totaux préparés à partir des deux laits (comparer [3 à 16]). Le dosage précis des différents acides polyinsaturés que contiennent les matières grasses respectives du lait de bufflonne et du lait de vache provenant d'animaux soumis à des conditions identiques de vie et d'alimentation a été effectué par spectrophotométrie ultraviolette [35].

Les résultats sont très voisins pour les diènes et les triènes, mais les proportions des tétraènes et des pentaènes sont plus élevées dans le lait de bufflonne que dans le lait de vache.

Le présent travail a pour objectif, d'une part, l'étude des acides gras présents à l'état de traces dans le lait de bufflonne et d'autre part la mise en évidence et l'identification de composés liposolubles également présents à l'état de traces et non encore reconnus dans le lait de bufflonne. Indépendamment de l'intérêt scientifique que présente une meilleure connaissance de la composition du lait de bufflonne, les résultats de l'étude envisagée, pourraient fournir des éléments de réponse au problème du contrôle de la pureté du lait de bufflonne. Le prix élevé de ce lait ( 3 fois plus cher que le lait 
de vache) constitue une incitation à la fraude dans les régions d'Italie où l'élevage du buffle est important.

\section{MATERIEL ET METHODES}

\section{Le fractionnement à l'urée. Principe et intérêt en analyse}

Le principe de la complexation à l'urée a été rappelé par Moreno et Roncero [30]. Les molécules d'urée pure cristallisent selon un schéma tétragonal compact; mais, lorsqu'elles forment des composés d'inclusion avec des substances comportant une chaîne normale aliphatique, les molécules d'urée construisent une charpente hexagonale dans laquelle s'insèrent les molécules du composé " hôte ". Une représentation schématique de cette charpente hexagonale est visible sur la figure 1. Les mesures des différents espacements sont visibles sur la figure 2 . La cellule unitaire de cette structure comporte 6 molécules d'urée occupant les arêtes d'un prisme et se disposant en spirale sur une distance de 11,1 $\dot{A}$. Les atomes d'oxygène de l'urée sont situés sur les arêtes des prismes et les groupements azotés s'étendent sur les surfaces planes qui séparent 2 prismes adjacents. Le diamètre intérieur des chenaux de la charpente hexagonale est de 5,2 À. Donc, seules les molécules dont la section atteint au maximum cette taille, peuvent former des composés d'inclusion. Une longueur minimale de chaîne est également requise pour former un complexe : 6 à 8 atomes de carbone sont nécessaires pour une cristallisation à température ambiante. La longueur maximale est de l'ordre de 48 atomes de carbone pour un hydrocarbure.

Les esters méthyliques d'acides gras à chaîne droite forment donc aisément des complexes avec l'urée, d'autant plus facilement que la chaîne est plus longue. Une seule ramification méthyle n'empêche pas la formation de complexe mais les molécules comportant d'importantes ramifications, constituées de plusieurs groupes méthyles rapprochés, ou de groupes $\mathrm{OH}$, et les molécules cycliques, ne forment pas de complexes.

La longueur de chaîne et l'insaturation jouent en sens inverse, c'est-à-dire que plus la chaîne est courte ou plus elle comporte des doubles liaisons, plus est difficile la complexation. La formation des complexes n'est jamais complète. Une certaine quantité résiduelle de composés reste dans la solution saturée d'urée. La disparition des composés n'est donc que progressive. Par ailleurs certains composés présents, qui ne forment pas de complexes, peuvent être entraînés ou adsorbés. Ces impuretés sont si fortement retenues par les cristaux qu'elles s'y maintiennent malgré les lavages répétés.

La technique de complexation à l'urée permet donc la concentration et parfois l'isolement de composés à structure particulière dans un milieu où les composés linéaires prédominent. 


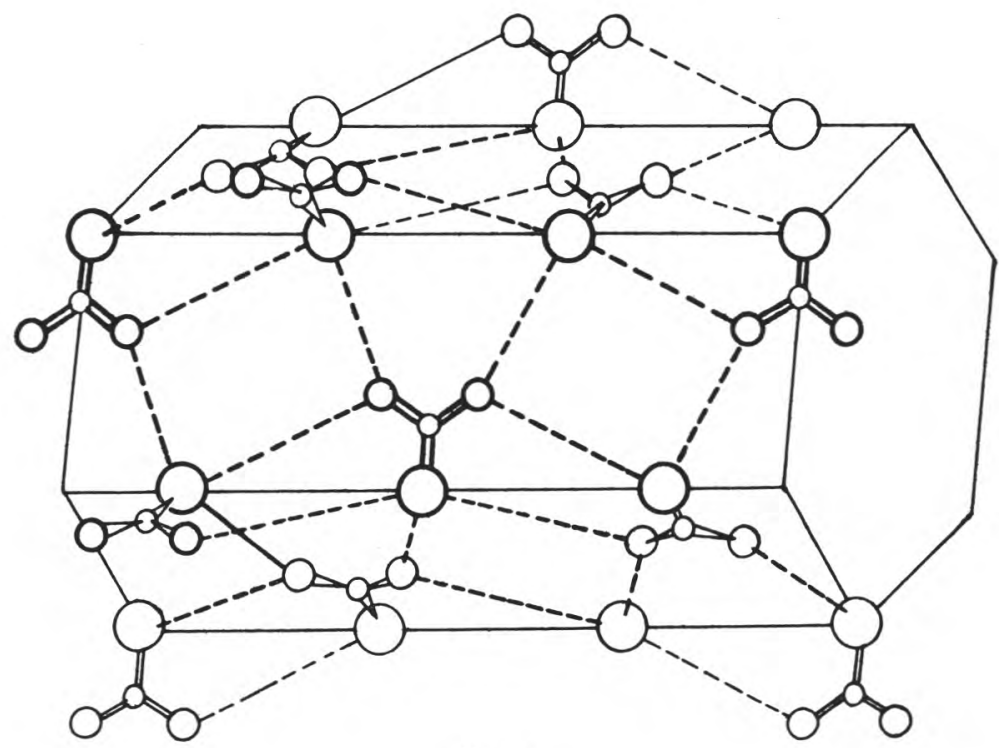

fig. 1

Disposition des molécules d'urée selon le système hexagonal. Les atomes d'oxygène (grands cercles) sont situés sur les arêtes du prisme. Les groupements $\mathrm{NH}_{2}$ (cercles moyens) s'étendent sur les surfaces planes.

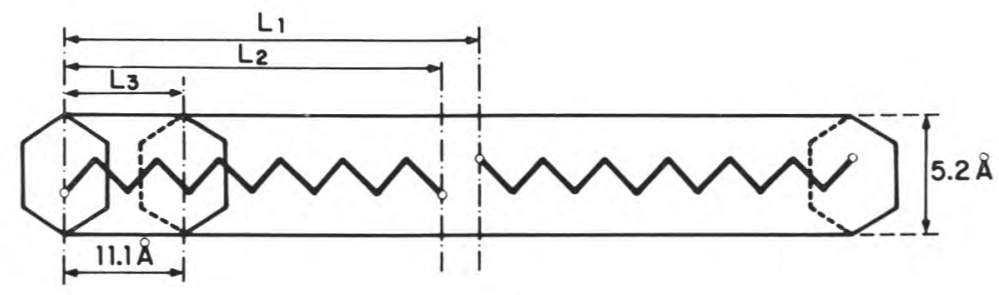

fig. 2

Représentation plane de l'association urée-molécule hote (30). $\mathrm{L}_{1}=$ longueur du chenal; $\mathrm{L}_{2}=$ longueur de la molécule incluse ; $\mathrm{L}_{3}=$ longueur d'une cellule unitaire.

\section{Application du fractionnement à l'urée à l'étude des composés liposolubles du lait de bufflonne}

Un prélèvement de 10 litres a été effectué sur le lait de mélange provenant d'un troupeau de bufflonnes comptant près de 500 ani- 
maux. Après écrémage du lait, la crème a été transformée en beurre. Après fusion et centrifugation de celui-ci la matière grasse obtenue a été séchée sur sulfate de sodium anhydre et filtrée.

Les esters méthyliques ont été préparés par transestérification à l'aide d'une solution méthanolique d'hydroxyde de potassium. Le mélange d'esters méthyliques contient donc également les composés liposolubles présents. Cet ensemble de composés a été soumis à un fractionnement par cristallisation à l'urée en suivant le protocole décrit par Iverson et al. [19].

Les esters méthyliques (50 g) préparés à partir de la matière grasse du lait de bufflonne sont dissous dans un grand volume $(800 \mathrm{ml})$ de méthanol. L'urée $(50 \mathrm{~g})$ est ajoutée. Le mélange est chauffé, agité jusqu'à dissolution de l'urée. La solution est maintenue 3 à $4 \mathrm{~h}$ à température ambiante et agitée par intermittence pendant la première demi-heure. Puis la solution est laissée au repos. Les cristaux formés sont recueillis par filtration sur verre fritté. Ils sont rincés à deux reprises avec un petit volume $(10 \mathrm{ml})$ de méthanol saturé d'urée. En vue de la récupération des esters ayant formé des complexes les cristaux sont additionnés d'une solution d'acide chlorhydrique à 1 p. $100(150 \mathrm{ml})$. Les esters libérés sont extraits à l'aide de $150 \mathrm{ml}$ d'éther de pétrole et $150 \mathrm{ml}$ d'oxyde d'éthyle. Une deuxième et une troisième extraction peuvent être effectuées. Les esters sont ensuite lavés à l'eau, séchés sur sulfate de sodium et recueillis après évaporation du solvant.

$\mathrm{Au}$ filtrat de la fraction 1 on ajoute $40 \mathrm{~g}$ d'urée et au besoin un peu de méthanol pour maintenir un volume constant de méthanol. Après chauffage, agitation, refroidissement, repos et filtration les esters inclus dans les cristaux sont recueillis comme précédemment.

Après obtention du dernier filtrat on ajoute à celui-ci environ $400 \mathrm{ml}$ d'acide chlorhydrique à 1 p. 100 et les esters accompagnés des composés liposolubles présents sont extraits puis recueillis après évaporation du solvant.

\section{Traitement complémentaire du dernier filtrat}

Les esters méthyliques contenus dans le dernier filtrat de cristallisation à l'urée ont été isolés des autres composés présents par une première chromatographie sur colonne de gel de silice. $500 \mathrm{mg}$ de résidu sec du filtrat en solution benzénique ont été déposés sur colonne $(50 \mathrm{~cm} \times 1,2 \mathrm{~cm})$ d'acide silicique. L'élution a été effectuée successivement par $100 \mathrm{ml}$ de benzène, $100 \mathrm{ml}$ d'un mélange de benzène et d'oxyde d'éthyle (9/1), $20 \mathrm{ml}$ d'oxyde d'éthyle pur et $20 \mathrm{ml}$ de méthanol ; onze fractions de $20 \mathrm{ml}$ ont été recueillies. La répartition des composés a été révélée par chromatographie sur couche mince. Les fractions contenant les esters méthyliques ont été réunies et soumises à un nouveau fractionnement par chromatographie sur 
colonne $(50 \mathrm{~cm} \times 1,2 \mathrm{~cm})$ d'acide silicique. L'élution a été effectuée à l'aide du mélange solvant éther de pétrole, oxyde d'éthyle (98/2); sept fractions de $40 \mathrm{ml}$ ont été recueillies. Les fractions 3 et 4 ont été étudiées.

\section{Analyse par chromatographie en phase gazeuse simple}

Les esters méthyliques régénérés à partir des différentes fractions cristallisant avec l'urée ont été analysés par chromatographie en phase gazeuse à l'aide d'un appareil Fractovap (modèle C, C. Erba, Milan), équipé d'une colonne capillaire en verre $(14 \mathrm{~m} \times 0,5 \mathrm{~mm}$ de diamètre intérieur). Les parois internes de la colonne étaient recouvertes d'une couche de graphite sur laquelle était déposée la phase stationnaire d'Apiezon L [34]. L'analyse était effectuée en programmation de température de 100 à $250^{\circ} \mathrm{C}$ avec un accroissement de température variable de 1 à $3,5^{\circ} \mathrm{C}$ par minute. A $150^{\circ} \mathrm{C}$, le nombre effectif de plateaux théoriques était de 20000 pour le méthylélaïdate. La surface des pics était calculée à l'aide d'un intégrateur HewlettPackard, modèle 3380 .

\section{Couplage chromatographie en phase gazeuse, spectrométrie de masse}

Les spectres de masse des esters méthyliques des acides gras ont été obtenus avec un appareil AEI-MS 30 à double faisceau couplé à un chromatographe Pye, modèle 104. Deux colonnes ont été utilisées, d'une longueur de $1,5 \mathrm{~m}$ et d'un diamètre intérieur de $0,25 \mathrm{~cm}$; elles étaient toutes deux remplies de diatomite CQ (100120 mesh) imprégnée au taux de 3 p. 100 soit d'Apiezon L soit de SE-30. Le gaz vecteur était de l'hélium (débit: $30 \mathrm{ml} / \mathrm{min}$ ). La température était programmée de 90 à $220^{\circ} \mathrm{C}$ pour l'analyse sur Apiezon L et de 120 à $290^{\circ}$ pour l'analyse sur SE-30, avec un même accroissement de température de $3^{\circ} \mathrm{C}$ par minute. La température de la source du spectromètre était $300^{\circ} \mathrm{C}$ et le potentiel de ionisation $70 \mathrm{eV}$. Les spectres ont été enregistrés au sommet de chaque pic chromatographique et en outre à différents niveaux de certains de ces pics. La vitesse d'enregistrement des pics était de 3 secondes par décade. L'analyse par couplage chromatographique en phase gazeuse et spectrométrie de masse a été appliquée aux différents mélanges d'esters provenant du fractionnement à l'urée et au dernier filtrat brut avant et après silylation.

\section{Identification des esters méthyliques d'acides gras par spectro- métrie de masse}

Les esters méthyliques des acides gras à chaîne linéaire sont caractérisés par un pic de base à m/e 74 dû au réarrangement de 
Mac Lafferty. Ils présentent aussi une fragmentation régulière de la chaîne aliphatique qui donne lieu aux ions du type $\left[\mathrm{CH}_{3} \mathrm{O} \underset{\mathrm{O}}{\mathrm{C}}\left(\mathrm{CH}_{2}\right)_{\mathrm{n}}\right]^{+}$: $87,101,115,129,143, \ldots \mathrm{M}^{+}-43, \mathrm{M}^{+}-29$; le pic ionique moléculaire est assez intense. Un pic à $\mathbf{M}^{+}-31$ est également présent. Le rapport de l'intensité des pics à $\mathbf{M}^{+}-29$ et $\mathbf{M}^{+}-31$ est, dans le cas de spectres des masses obtenus à partir des acides gras à chaîne linéaire, toujours inférieur à 1 .

Les esters méthyliques des acides gras à chaîne ramifiée de la série iso et anteiso, bien séparés dans les conditions de l'analyse chromatographique, sont aussi bien différenciés par spectrométrie de masse. Ils présentent une fragmentation similaire à celle des acides gras à chaîne linéaire mais comportent en outre certains pics induits par la ramification. En particulier la fragmentation en position $\alpha$ suivie de la perte de méthanol et d'eau conduit à la formation des pics du type $\mathrm{M}^{+}-(\mathrm{R}+32)$ et $\mathrm{M}^{+}-(\mathrm{R}+32+18)$ où $\mathrm{R}$ est le groupement alkyl sur le carbone tertiaire [37]. Ces pics ne sont pas très intenses. Pour les acides gras de la série antéiso le rapport intensité du pic $\mathrm{M}^{+}-29$ /intensité du pic $\mathrm{M}^{+}-31$ constitue un élément de diagnostic car il devient dans ce cas supérieur à l'unité [1].

Les esters méthyliques des acides gras multiramifiés donnent des spectres de masse à partir desquels il est possible de déduire l'emplacement des ramifications. En effet les fragmentations en position $\alpha$ du carbone tertiaire deviennent prépondérantes.

\section{RESULTATS}

\section{Le fractionnement à l'urée}

Un fractionnement effectué sur $50 \mathrm{~g}$ d'esters méthyliques totaux préparés directement à partir de la matière grasse de lait de bufflonne a permis d'obtenir successivement cinq fractions cristallisées correspondant à :

- fraction 1: 4,0 $\mathrm{g}$;

- fraction 2: 9,7 g ;

- fraction $3: 10,7 \mathrm{~g}$;

- fraction 4: 16,6 g ;

- fraction 5: 4,5 g.

Le dernier filtrat après cristallisation contenait un résidu de 0,9 g constitué par un mélange d'esters méthyliques et de composés liposolubles divers.

Le fractionnement supplémentaire de la fraction 1 a conduit à l'obtention d'une fraction cristallisée (1 a) regroupant 1,1 g d'esters méthyliques et à un filtrat regroupant 2,6 d'esters méthyliques (1 b). 


\section{Purification des esters méthyliques du dernier filtrat de cristal- lisation à l'urée}

La chromatographie en couche mince du dernier filtrat de cristallisation à l'urée montre la complexité de cette fraction (fig. 3). Celle-ci renferme des hydrocarbures, des esters méthyliques, des stérols, etc. Des composés très polaires visibles dans le filtrat total restent au point de départ et n'ont pas été recueillis. Les composés présents

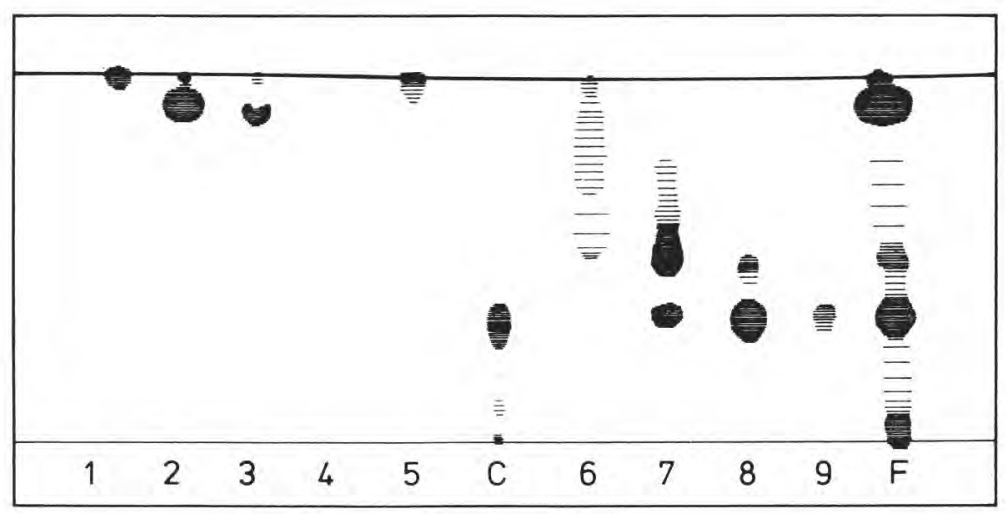

fig. 3

Chromatographie en couche mince des composés présents dans les différentes fractions collectées lors de la chromatographie sur colonne du filtrat brut obtenu en fin de fractionnement à l'urée. Les fractions 2 et 3 contiennent les esters méthyliques. $\mathrm{C}=$ cholestérol ; $\mathrm{F}=$ filtrat final du fractionnement à l'urée.

dans la fraction 2 et 3 (essentiellement des esters méthyliques) ont été rassemblés et chromatographiés à nouveau. Les fractions 3 et 4 , rassemblées, résultant de cette nouvelle chromatographie ont été utilisées lors de l'étude par spectrométrie de masse. Les résultats pondéraux obtenus au cours des étapes de purification des esters méthyliques du filtrat sont indiqués dans le tableau 1.

\section{Identification des esters méthyliques}

a) Identification des esters méthyliques dans les fractions résultant du fractionnement à l'urée

La formation des complexes à l'urée est fonction de la structure des acides gras et de leur poids moléculaire. Chaque fraction est 


\section{TABLEAU 1}

Déroulement des étapes de purification des esters méthyliques du filtrat obtenu en fin de fractionnement à l'urée

\begin{tabular}{l|c|c}
\hline \multicolumn{2}{c}{ Purification première étape } \\
\hline \multirow{3}{*}{ Eluant } & $\begin{array}{c}\text { Fractions } \\
(20 \mathrm{ml})\end{array}$ & $\begin{array}{c}\text { Poids de l'extrait } \\
(\mathrm{mg})\end{array}$ \\
\hline \multirow{3}{*}{ Benzène } & & \\
& 1 & 6 \\
& 2 & 178 \\
Benzène-oxyde d'éthyle $(9 / 1)$ & 3 & 55 \\
& 4 & 1 \\
& 5 & 1 \\
& 6 & 5 \\
Oxyde d'éthyle & 7 & 34 \\
Méthanol & 8 & 4 \\
& 9 & 22 \\
\hline
\end{tabular}

Purification deuxième étape

\begin{tabular}{l|c|c}
\hline Eluant & $\begin{array}{c}\text { Fractions } \\
(40 \mathrm{ml})\end{array}$ & $\begin{array}{c}\text { Poids de l'extrait } \\
(\mathrm{mg})\end{array}$ \\
\hline Ether de pétrole oxyde d'éthyle & 1 & 1 \\
$(98 / 2)$ & 2 & 8 \\
& 3 & 83 \\
& 4 & 100 \\
5 & 28 \\
& 6 & 2 \\
& 7 & 1 \\
\hline
\end{tabular}

donc enrichie en un type particulier d'esters méthyliques. Ceci facilite la mise en évidence des esters méthyliques d'acides gras présents à l'état de traces et leur identification par spectrométrie de masse.

Sur la figure 4 est reproduit le chromatogramme obtenu à partir des esters totaux du lait de bufflonne. La phase stationnaire étant de l'Apiezon L, les acides insaturés précèdent les acides saturés à même nombre d'atomes de carbone. L'analyse globale des acides gras du lait de bufflonne est tout à fait similaire à l'analyse globale des esters méthyliques préparés à partir de la matière grasse du lait de vache. En particulier l'acide monoène trans à 18 atomes de carbone est également présent. 


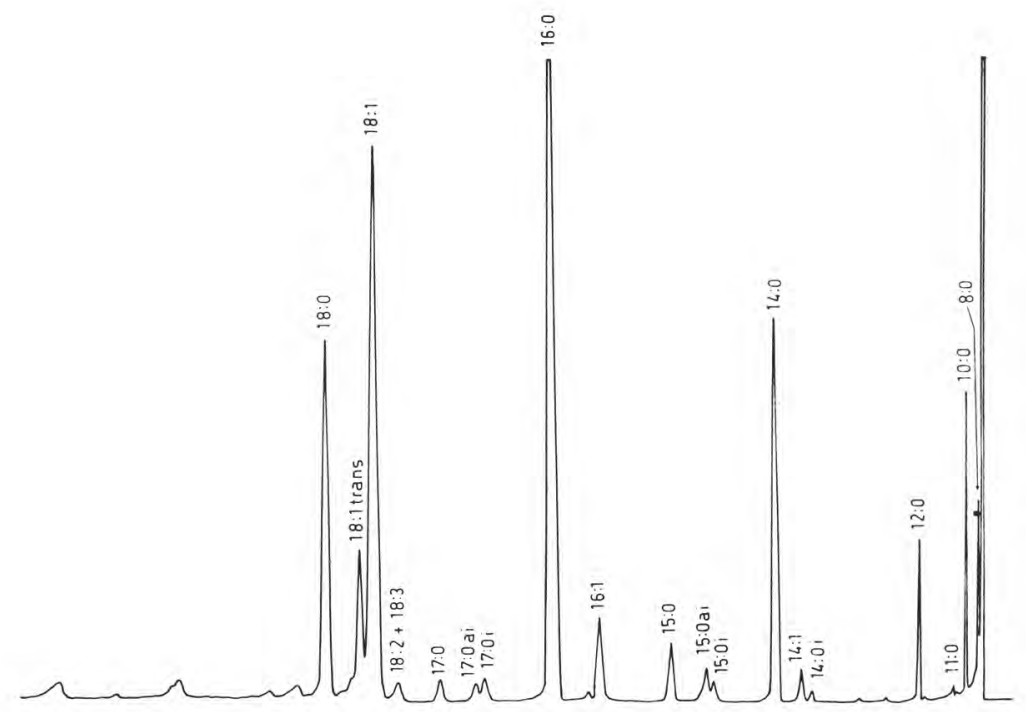

fig. 4

Analyse par chromatographie en phase gazeuse des esters méthyliques totaux de la matière grasse du lait de bufflonne. Colonne capillaire d'Apiezon L. Programmation de la température de $120^{\circ} \mathrm{C}$ à $200^{\circ} \mathrm{C}$ avec un accroissement de $1,25^{\circ} \mathrm{C} / \mathrm{min}$.

Les analyses des esters méthyliques de la fraction recristallisée (1 a) et du filtrat ( $1 \mathrm{~b})$ provenant de la fraction 1 résultant du fractionnement à l'urée, sont présentés sur les figures $5 \mathrm{a}$ et $5 \mathrm{~b}$. Les chromatogrammes présentés ont été obtenus au cours d'une analyse par chromatographie en phase gazeuse sur colonne capillaire d'Apiezon L, mais, lors de l'identification des acides, effectuée par couplage chromatographie en phase gazeuse-spectrométrie de masse, une colonne courte contenant du SE-30 comme phase stationnaire a été utilisée. Les acides à très longue chaîne (19 à 28 atomes de carbone) sont nettement visibles sur les chromatogrammes présentés sur les figures $5 \mathrm{a}$ et $5 \mathrm{~b}$ alors qu'ils n'apparaissent pas sur un chromatogramme obtenu à partir des esters méthyliques totaux. Les acides gras saturés à longue chaîne sont en proportion plus élevés dans la fraction cristallisée (a) que dans le filtrat (b). Au total les acides à longue chaîne représentent une proportion importante des acides gras rassemblés dans la première fraction récoltée lors du fractionnement par complexation à l'urée (tableau 2). 


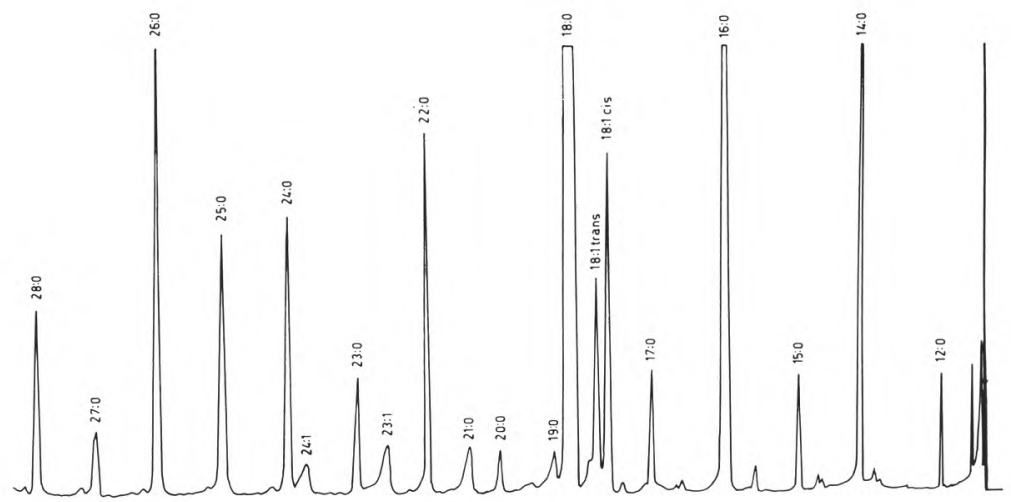

fig. $5 a$

Analyse par chromatographie en phase gazeuse des esters méthyliques préparés à partir de la fraction recristallisée $(1 \mathrm{a})$ provenant de la fraction 1 (fractionnement à l'urée). Colonne capillaire d'Apiezon L. Programmation de température de 120 à $240^{\circ} \mathrm{C}$ avec un accroissement de $1,25^{\circ} \mathrm{C} / \mathrm{min}$.

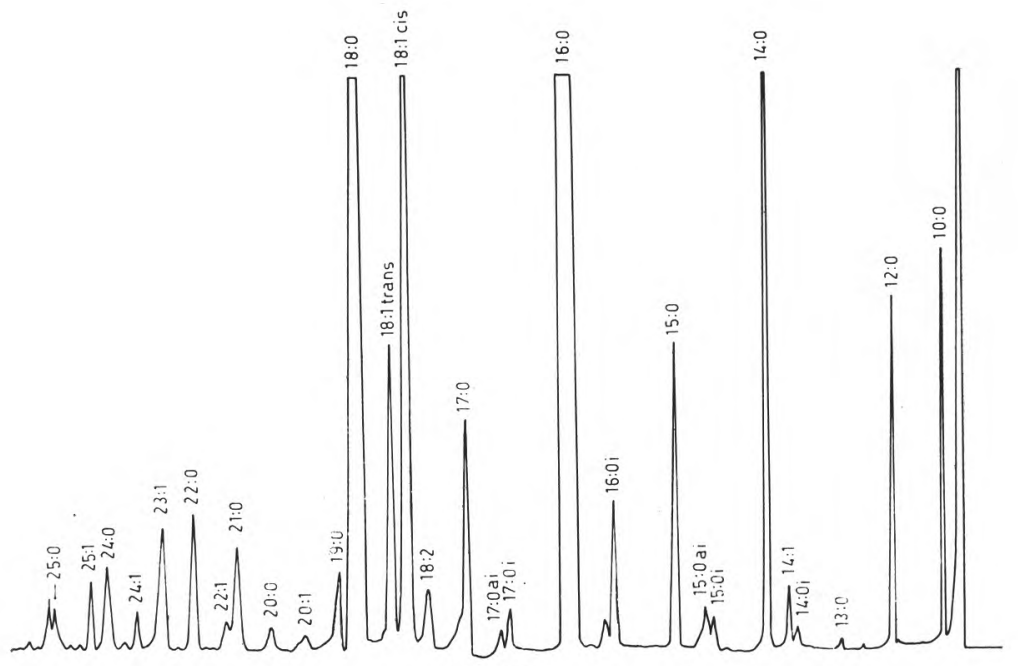

fig. $5 b$

Analyse par chromatographie en phase gazeuse du filtrat $1 \mathrm{~b}$ provenant de la fraction 1 (fractionnement à l'urée). Colonne capillaire Apiezon L. Programmation de température de 100 à $200^{\circ} \mathrm{C}$ avec un accroissement de $1,25^{\circ} \mathrm{C} / \mathrm{min}$. 


\section{TABLEAU 2}

Proportions relatives des acides gras saturés linéaires dans les fractions 1 résultant du fractionnement à l'urée des esters méthyliques totaux du lait de vache et du lait de bufflonne (résultats exprimés en p. 100 des esters totaux des fractions 1)

\begin{tabular}{c|c|c}
\hline Acide & Bufflonne & Vache * \\
\cline { 2 - 2 } $19: 0$ & 0,6 & 1,9 \\
$20: 0$ & 2,7 & 2,5 \\
$21: 0$ & 2,4 & 1,3 \\
$22: 0$ & 6,0 & 3,6 \\
$23: 0$ & 5,1 & 2,4 \\
$24: 0$ & 8,8 & 3,3 \\
$25: 0$ & 1,8 & 0,6 \\
$26: 0$ & 4,0 & 4,2 \\
$27: 0$ & traces & 0,1 \\
$28: 0$ & 1,0 & 3,0 \\
& & \\
\hline
\end{tabular}

* Pour comparaison: ces données proviennent d'un travail analogue poursuivi sur la matière grasse du lait de vache, non publié.

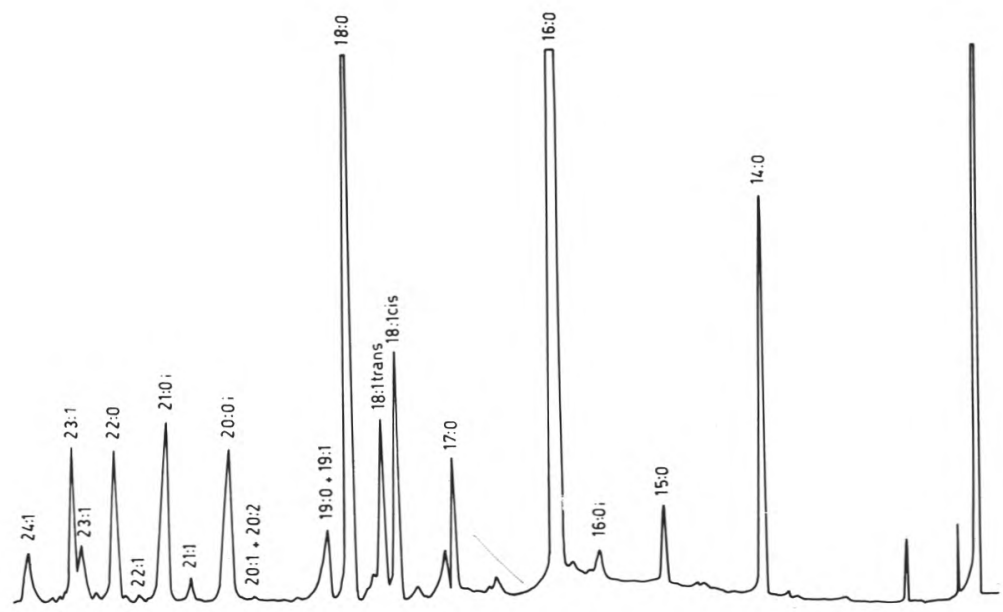

fig. 6

Analyse par chromatographie en phase gazeuse de la fraction 2 . Colonne capillaire d'Apiezon L. Programmation de température de 120 à $250^{\circ} \mathrm{C}$ avec un accroissement de $1,5^{\circ} \mathrm{C} / \mathrm{min}$. 
La figure 6 reproduit le chromatogramme obtenu à partir de la fraction 2 du fractionnement à l'urée. On y identifie des acides à longue chaîne, monoènes et ramifiés, qui n'apparaissent pas ou n'apparaissent qu'à peine dans la fraction précédente.

Dans la fraction 3 (fig. 7) les acides saturés à relative courte chaîne (16 et 14 atomes de carbone) sont visibles et accompagnent l'acide monoène trans à 18 atomes de carbone. Celui-ci représente 7,5 p. 100 des acides gras totaux.

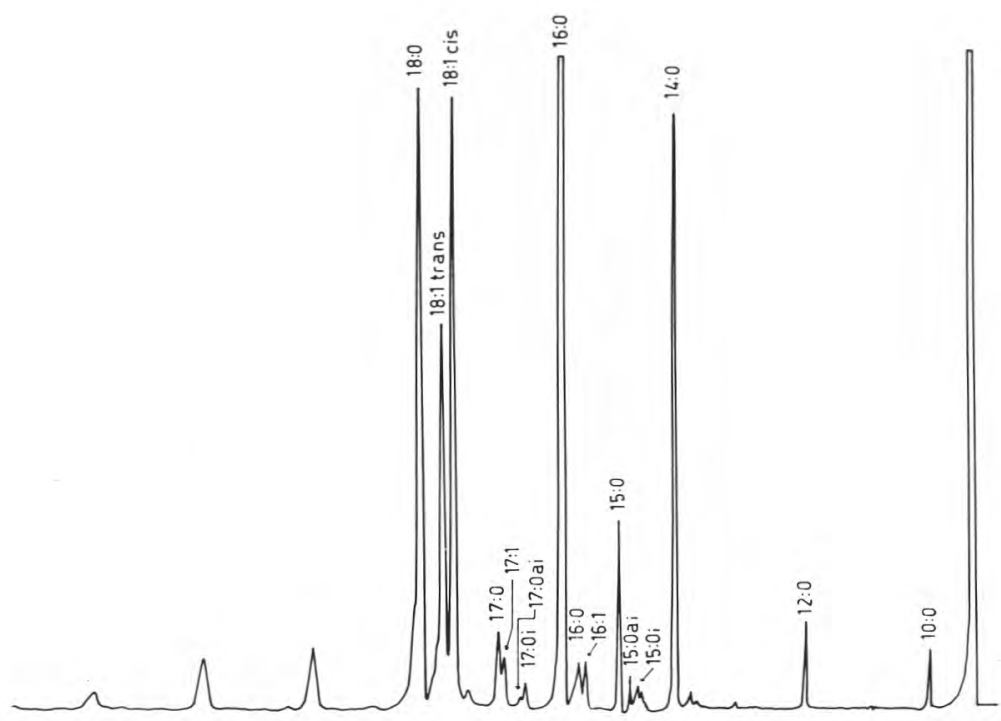

fig. 7

Analyse par chromatographie en phase gazeuse de la fraction 3. Colonne capillaire Apiezon L. Programmation de la température de 100 à $160^{\circ} \mathrm{C}$ avec un accroissement de $3,5^{\circ} \mathrm{C} / \mathrm{min}$.

Dans la fraction 4 (fig. 8) l'acide oléique prédomine à côté de l'acide monoène à 16 atomes de carbone. Dans cette fraction 4 réapparaissent des acides monoènes à longue chaîne (à 22 et à 23 atomes de carbone) déjà signalés dans les fractions 1 et 2 , mais pratiquement absents dans la fraction 3. Ces acides possèdent vraisemblablement une structure particulière qui retarde leur complexation avec l'urée.

Les acides ramifiés à moyenne chaîne (comptant 17, 15 et 14 atomes de carbone) apparaissent dans la fraction 5 (fig. 9) accompagnant les acides monoènes à même nombre d'atome de carbone. Les acides polyinsaturés (18:2 et 18:3) se rassemblent dans cette 


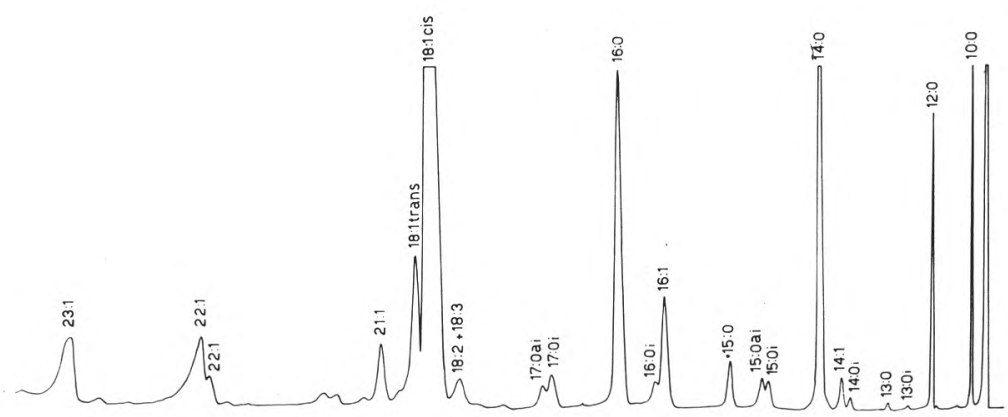

fig. 8

Chromatographie en phase gazeuse des esters méthyliques de la fraction 4. Colonne capillaire d'Apiezon L. Programmation de température de 110 à $230^{\circ} \mathrm{C}$ avec un accroissement de $3^{\circ} \mathrm{C} / \mathrm{min}$.

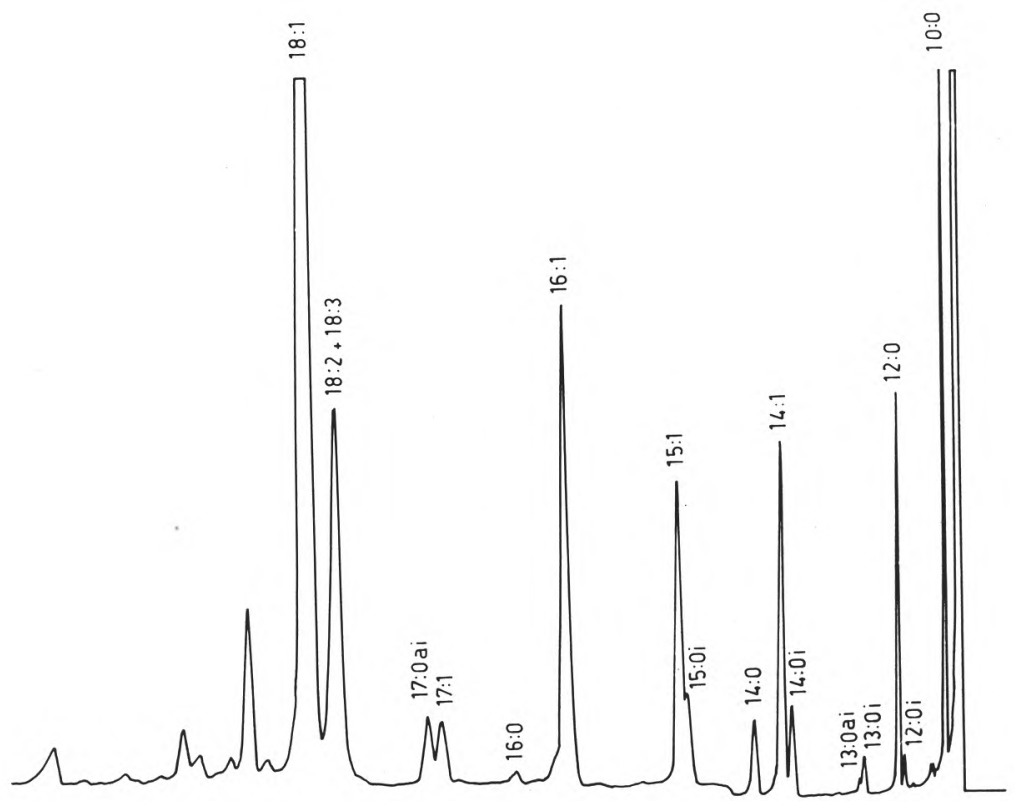

fig. 9

Chromatographie en phase gazeuse des esters méthyliques de la fraction 5. Colonne capillaire d'Apiezon L. Programmation de température de 100 à $200^{\circ} \mathrm{C}$ avec un accroissement de $3^{\circ} \mathrm{C} / \mathrm{min}$. 
fraction. La proportion de l'acide linoléique est voisine de 8 p. 100 des acides gras de la fraction 5 , soit près de 10 fois supérieure à la proportion qui est observée pour ce même acide dans une analyse globale des acides gras de lait de bufflonne.

L'analyse directe par couplage chromatographie en phase gazeuse et spectrométrie de masse du dernier filtrat brut a permis de mettre en évidence les acides monoènes à 14,12 et 10 atomes de carbone et l'acide ramifié iso comptant 10 atomes de carbone.

b) Identification des esters méthyliques dans le filtrat après purification par chromatographie sur colonne

Analysée par chromatographie en phase gazeuse, la fraction 3, résultant de la deuxième purification du filtrat final sur colonne d'acide silicique, conduit au chromatogramme présenté sur la figure 10. Sont mis en évidence 5 composés qui, dans l'ordre d'élution

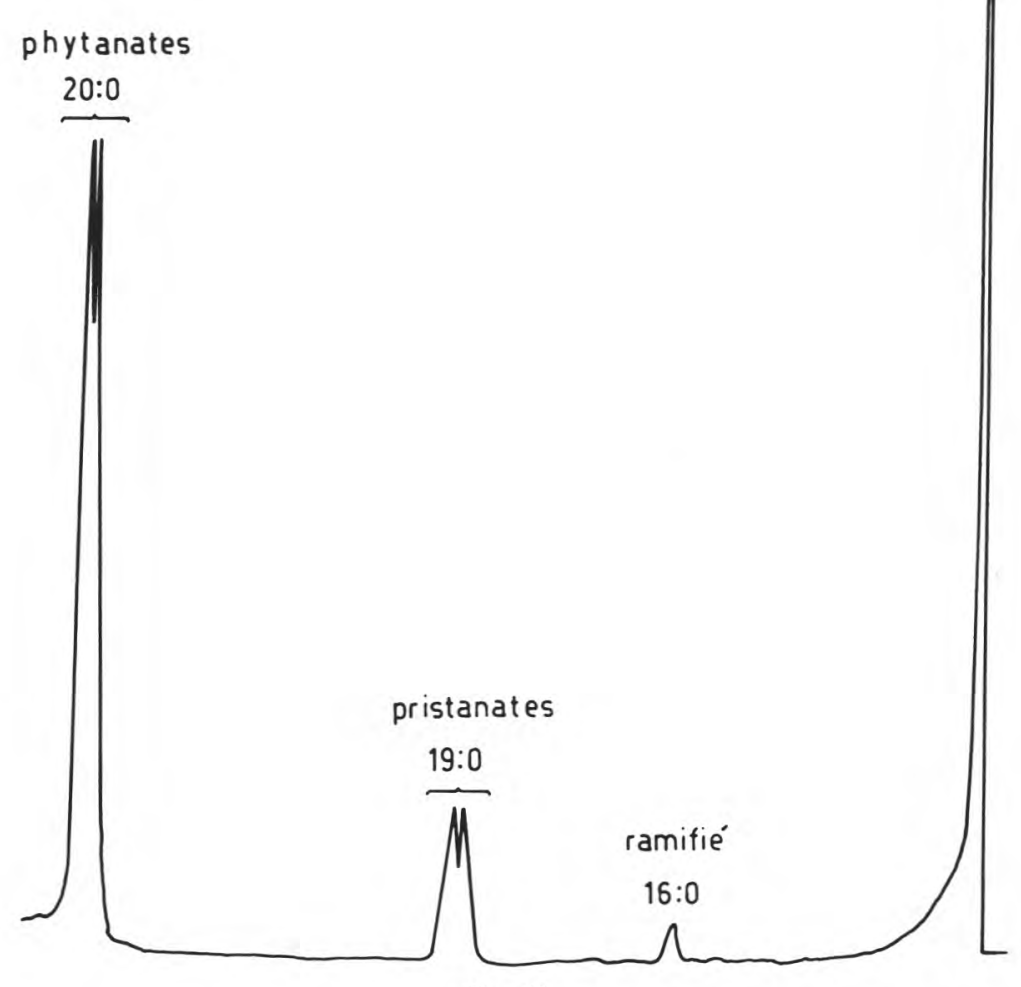

fig. 10

Analyse par chromatographie en phase gazeuse des esters méthyliques saturés à chaîne ramifiée isolés par chromatographie préparative sur gel de silice à partir du filtrat final du fractionnement à l'urée. Colonne capillaire d'Apiezon L. Programmation de température de 90 à $200^{\circ} \mathrm{C}$ avec un accroissement de $1,25^{\circ} \mathrm{C} / \mathrm{min}$. 
sur colonne capillaire d'Apiezon L présentent des masses moléculaires de 270 (un composé), 312 ( 2 composés) et 326 ( 2 composés). L'analyse par spectrométrie de masse a permis d'identifier le premier pic : il s'agit du 4,8,12-triméthyltridécanoate de méthyle. Les deux pics adjacents en milieu de figure correspondent aux esters méthyliques respectivement des deux formes isomères LDD et DDD de l'acide 2, 6, 10,14-tétraméthylpentadécanoïque ou acide pristanique. Les deux derniers pics correspondent, de la même façon, aux esters méthyliques respectivement des deux formes isomériques de l'acide $3,7,11$, 15-tétraméthylhexadécanoïque. Ces 5 acides ramifiés représentent ensemble 0,17 p. 100 des acides gras totaux du lait de bufflonne.

Connaissant les proportions relatives des différentes fractions obtenues au cours du fractionnement à l'urée et connaissant également la répartition pondérale des esters méthyliques dans ces fractions, il a été possible de calculer une composition type des acides

\section{TABLEAU 3}

Composition en acides gras de la graisse du lait de bufflonne soumis à l'analyse

\begin{tabular}{|c|c|c|c|c|c|}
\hline Acide & p. 100 & $\mathrm{M}^{+}$ & Acide & p. 100 & $\mathrm{M}^{+}$ \\
\hline $4: 0$ & 3,4 & 102 & $18: 2$ & 0,8 & 294 \\
\hline $6: 0$ & 2,5 & 130 & $18: 3$ & 1,1 & 292 \\
\hline 8: 0 & 1,2 & 158 & 19:0 & 0,03 & 312 \\
\hline $10: 0$ & 1,9 & 186 & $19: 0(2,6,10.14$ TMPD $)$ & 0,019 & 312 \\
\hline $10: 0$ iso & 0,012 & 186 & 19:1 & 0,07 & 310 \\
\hline $10: 1$ & 0,17 & 184 & $19: 1$ & 0,007 & 310 \\
\hline 11:0 & 0,16 & 200 & $20: 0$ & 0,4 & 326 \\
\hline $12: 0$ & 3,19 & 214 & $20: 0$ iso & 0,3 & 326 \\
\hline $12: 1$ & 0,01 & 212 & $20: 0(3,7,11,15$ TMHD $)$ & 0,14 & 326 \\
\hline 13:0 & 0,19 & 228 & $20: 1$ & 0,1 & 324 \\
\hline $13: 0$ iso & 0,32 & 228 & $20: 2$ & 0,017 & 322 \\
\hline $13: 0 \mathrm{ai}$ & 0,14 & 228 & $21: 0$ & 0,087 & 340 \\
\hline $14: 0$ & 12,22 & 242 & $21: 0$ iso & 0,002 & 340 \\
\hline $14: 0$ iso & 0,006 & 242 & $21: 1$ & 0,013 & M-32 \\
\hline $14: 1$ & 0,5 & 240 & $21: 1$ & 0,004 & $n$ \\
\hline 15:0 & 0,9 & 256 & $22: 0$ & 0,2 & 354 \\
\hline $15: 0$ iso & 0,8 & 256 & $22: 0$ iso & 0,006 & 354 \\
\hline $15: 0$ ai & 0,8 & 256 & $22: 1$ & 0,014 & M-32 \\
\hline $15: 1$ & 0,03 & 254 & $22: 1$ & 0,006 & " \\
\hline $16: 0$ & 22,2 & 270 & 23:0 & 0,1 & 368 \\
\hline $16: 0$ iso & 0,3 & 270 & $23: 0$ iso & 0,002 & 368 \\
\hline $16: 0(4,8,12$ TMTD $)$ & 0,006 & 270 & $23: 1$ & 0,034 & M-32 \\
\hline $16: 1$ & 2,5 & 268 & $23: 1$ & 0,008 & " \\
\hline $17: 0$ & 0,7 & 284 & $24: 0$ & 0,3 & 382 \\
\hline $17: 0$ iso & 0,3 & 284 & $24: 1$ & 0,004 & M-32 \\
\hline $17: 0 \mathrm{ai}$ & 0,3 & 284 & $25: 0$ & 0,003 & 396 \\
\hline $17: 1$ & 0,4 & 282 & $25: 1$ & 0,008 & M-32 \\
\hline 18:0 & 11.7 & 298 & $26: 0$ & 0,15 & 410 \\
\hline $18: 0$ iso & 0,016 & 298 & $27: 0$ & 0,001 & 424 \\
\hline $18: 1$ cis & 21,4 & 296 & $28: 0$ & 0,010 & 438 \\
\hline $18: 1$ trans & 7,5 & 296 & & & \\
\hline
\end{tabular}


gras totaux du lait de bufflonne. Cette composition est présentée dans le tableau 3 ; la masse de l'ion moléculaire est également indiquée pour chaque acide gras. Les proportions relatives des acides comptant 4 à 10 atomes de carbone ont été déterminées à partir d'une analyse globale des acides gras. Au total 61 acides gras ont été identifiés dans la matière grasse du lait de bufflonne sans tenir compte des isomères de position qui n’ont pas été étudiés.

\section{Identification des composés mineurs liposolubles autres que les acides gras}

Lors de l'analyse du filtrat brut par chromatographie en phase gazeuse sur la colonne des SE-30 montée dans l'appareil Pye couplé au spectromètre de masse, on a obtenu l'élution de plusieurs pics au-delà du pic de l'ester méthylique de l'acide saturé comptant 20 atomes de carbone. Un chromatogramme partiel est présenté sur la figure 11 .

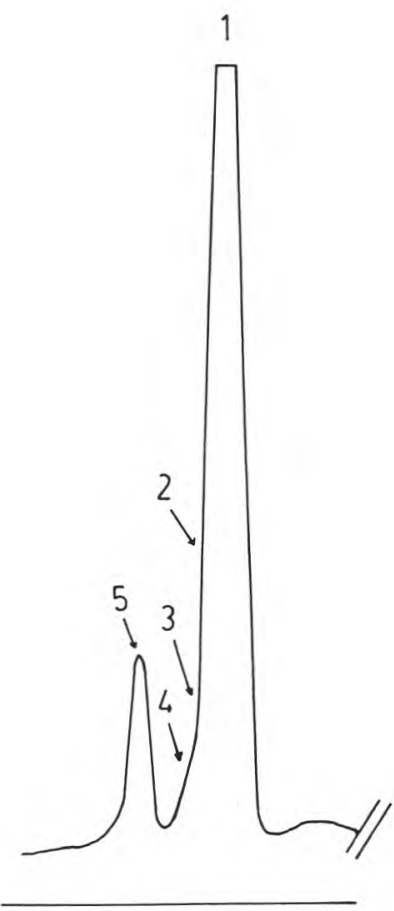

fig. 11

Chromatogramme partiel (courant ionique total) obtenu lors de l'analyse par couplage chromatographie en phase gazeusespectrométrie de masse du filtrat brut final résultant du fractionnement à l'urée. Phase stationnaire: SE-30. Programmation de température: 120 à $290^{\circ} \mathrm{C}$ avec un accroissement de $3^{\circ} \mathrm{C} / \mathrm{min}$. 
Le pic du cholestérol [1] est bien individualisé. Il est caractérisé par un ion moléculaire à $\mathrm{m} / \mathrm{e} 386$. Sur la descente du pic du cholestérol on relève la présence de plusieurs composés différents dénommés 2,3,4 et révélés par leur spectre de masse. Le premier de ces composés [2] a un pic moléculaire $\mathbf{M}^{+}$à $\mathrm{m} / \mathrm{e} 384$. Le pic de base est à $\mathrm{m} / \mathrm{e} 271$. Les autres pics importants sont à $\mathrm{m} / \mathrm{e} 369\left(\mathrm{M}^{+}-15\right.$; 45 p. 100), 384 (27 p. 100), 272 (15 p. 100), 300 (11 p. 100) et 299 (10 p. 100). L'existence d'un pic à $\mathrm{m} / \mathrm{e} 314\left(\mathrm{M}^{+}-70\right)$ indique la présence d'une double liaison en position 24 par effet de la coupure qui se produit en position $\beta$ de la double liaison. Ce spectre est voisin d'un spectre reporté dans "Eighth peak index of mass spectra " [13] (vol. 1, p. 513 ou dans EPA/NIH mass spectral data base [15], p. 384) dont les caractéristiques sont les suivantes: pic moléculaire à $\mathrm{m} / \mathrm{e} 384$, pic de base à 271 et pics principaux à $272(31 \mathrm{p} .100)$, 369 (31 p. 100 ), 384 (30 p. 100), 300 (26 p. 100), 299 (19 p. 100), 95 (17 p. $100)$ et 69 ( 16 p. 100). Le composé correspondant, de formule brute $\mathrm{C}_{27} \mathrm{H}_{44} \mathrm{O}$ est le cholesta-5,24-dièn-3-ol $(3 \beta)$ ou "desmostérol ". Le composé dénommé 3 (sur la descente du pic du cholestérol) présente un pic moléculaire à m/e $396(47 \mathrm{p} .100)$. Les autres pics significatifs, s'ajoutant au pic de base à $\mathrm{m} / \mathrm{e} 281$, apparaissent à $\mathrm{m} / \mathrm{e} 324\left(\mathrm{M}^{+}\right.$ $-72 ; 50$ p. 100), $325\left(\mathrm{M}^{+}-71 ; 15\right.$ p. 100), $363\left(\mathrm{M}^{+}-15-18 ; 25\right.$ p. 100), $378\left(\mathbf{M}^{+}-18 ; 34\right.$ p. 100 et $381\left(\mathbf{M}^{+}-15 ; 26\right.$ p. 100). Il semble que ce produit appartienne à la classe des stéroïdes insaturés avec un groupement cétone en position 3 .

Le spectre de masse du pic 4 individualisé sur le chromatogramme de la figure 11 conduit à un produit de masse moléculaire 426 (28 p. 100) dont le pic de base est à m/e $411\left(\mathrm{M}^{+}-15 ; 100 \mathrm{p} .100\right)$. Les autres pics sont à $\mathrm{m} / \mathrm{e} 393\left(\mathrm{M}^{+}-33 ; 40 \mathrm{p} .100\right)$, à $273\left(\mathrm{M}^{+}-\right.$ chaîne latérale $+42+15 ; 11$ p. 100$)$, à $259\left(\mathrm{M}^{+}\right.$- chaîne latérale $+42+15+18 ; 11$ p. 100 ). Un pic métastable correspondant à la transition $411 \rightarrow 393$ est à $\mathrm{m} / \mathrm{e} 375,8$. Ce composé présente la même fragmentation que le produit de référence: «le lanostérol ». Un spectre similaire à celui décrit est détaillé dans la référence suivante: EPA/NIH mass spectral data base, p. 3265 [15].

Le lanostérol a pour formule globale $\mathrm{C}_{30} \mathrm{H}_{50} \mathrm{O}$, son nom chimique est : Lanosta-8,24-dien-3-ol (3 $\beta$ ).

Le pic 5, bien séparé sur le chromatogramme présenté sur la figure 11 est un mélange de 2 produits à m/e 426 et 424 , le pic de base est à m/e 232 et d'autres pics importants sont rencontrés à $\mathrm{m} / \mathrm{e} 217,268$ et 253 . La fragmentation de ces deux produits est totalement différente de celle du lanostérol. Le filtrat brut dans lequel ont été identifiés certains composés liposolubles a été soumis à silylation. Un nouveau chromatogramme (fig. 12) a été obtenu à partir du filtrat ainsi traité. Une partie de ce chromatogramme est présentée sur la figure 12 . 


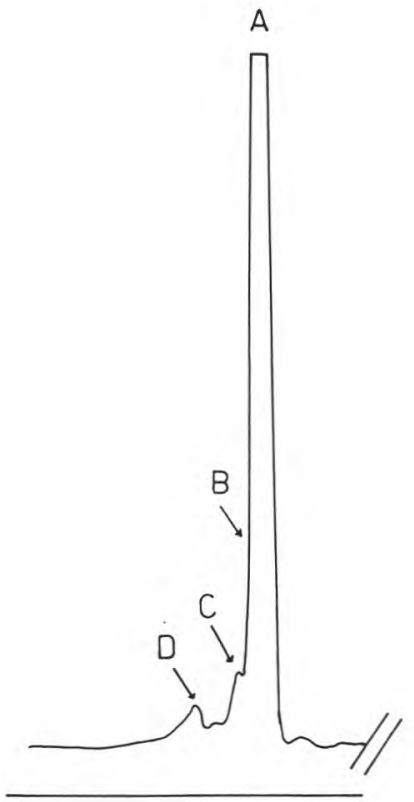

fig. 12

Même analyse qu'en figure 11, mais après silylation du filtrat brut.

Après l'élution du cholestérol, A (bien reconnaissable à son pic moléculaire de m/e 458 et aux autres pics tels que le 129 [100 p. 100]), apparaît le composé B à m/e 456 (soit $384+72$ ), triméthylsilyl dérivé du desmostérol. Le pic de base est à $\mathrm{m} / \mathrm{e} 129$. Des pics à $\mathrm{m} / \mathrm{e} 247$ (15 p. 100), 255 (14 p. 100), 275 (10 p. 100), 327 (77 p. 100), 351 (27 p. $100), 366(36 \mathrm{p} .100)$ et $441(7 \mathrm{p} .100)$ sont aussi présents. Le pic à $\mathrm{m} / \mathrm{e} 255\left(\mathrm{M}^{+}-90-111\right)$ permet de localiser les deux doubles liaisons, une dans le moyen stéroïde, l'autre dans la chaîne latérale. Dans le cas du triméthylsilyl dérivé du 7-déhydro-cholestérol $\left(\mathrm{M}^{+}=456\right)$ dans lequel les deux doubles liaisons sont localisées dans le noyau stéroïdique on observerait au lieu du pic à m/e 255 comme dans le cas du desmostérol, un pic à $\mathrm{m} / \mathrm{e} 253\left(\mathrm{M}^{+}\right.$- 90-113) parce que la perte de la chaîne latérale donne lieu à un fragment saturé avec deux unités de masse en plus [9]. Mais en outre apparaît un composé mineur, $\mathrm{C}$, de masse moléculaire $\mathrm{m} / \mathrm{e} 458$ dont le spectre possède les caractéristiques suivantes : 255 (100 p. 100), 213 (50 p. 100), 229 (40 p. 100), 107 (30 p. 100), 459 (27 p. 100), 147 (26 p. 100) et 353 (20 p. 100). Ce spectre correspond au dérivé triméthylsilylé du lathostérol $(386+72)$ qui a la formule brute $\mathrm{C}_{27} \mathrm{H}_{46} \mathrm{O}$ (Eight peak index of mass spectra, vol. I, p. 550) [13]. 
Le produit D a le même poids moléculaire que le lanostérol, c'est-à-dire 426, mais ce composé ne forme pas le dérivé triméthylsilylé. L'identification de ce composé est en cours.

\section{DISCUSSION}

\section{Appréciation du fractionnement à l'urée}

Cette méthodologie simple permet de concentrer les acides gras dans l'une ou l'autre des fractions obtenues. Ceci facilite l'identification de ces acides par spectrométrie de masse lorsque celle-ci est utilisée avec la chromatographie en phase gazeuse. La méthodologie utilisée permet de rassembler dans le filtrat, les constituants liposolubles qui ne forment pas de complexe avec l'urée, sans risque de dénaturation comme cela peut se produire lorsqu'une saponification intermédiaire intervient. Le fractionnement par complexation à l'urée a une portée générale et peut être utilisé dans de nombreuses occasions. Dans le cas présent le fractionnement à l'urée a permis de mettre en évidence les acides saturés à très longue chaîne qui se concentrent dans la première fraction cristallisée. Dans le tableau 2 ont été portés, à côté des résultats concernant la matière grasse du lait de bufflonne, les résultats obtenus après application de cette même méthode de fractionnement à l'urée au mélange d'esters méthyliques préparés à partir de la matière grasse de lait de vache. Il est possible d'envisager une application de cette observation au contrôle de pureté du lait de bufflonne. Le rapport 24:0/26:0 (supérieur à 2,0 pour le lait de bufflonne mais inférieur à 1,0 pour le lait de vache) pourrait être le critère retenu.

\section{Etude comparée des proportions relatives des acides gras dans le lait de bufflonne et le lait de vache}

La matière grasse du lait de bufflonne renferme les mêmes acides gras que la matière grasse du lait de vache. L'acide vaccénique (18:1 trans) est présent à un taux voisin de 7,5 p. 100 , c'est-à-dire à un taux équivalent à celui trouvé dans le lait de vache en période de pâturage [24]. Bien que la proportion d'acide linoléique déterminée par chromatographie en phase gazeuse soit relativement imprécise, la valeur obtenue, - un peu plus faible que celle trouvée dans les mêmes conditions, pour le lait de vache, - confirme les résultats antérieurs de Ramamurthy et Narayanan [35]. Le fractionnement à l'urée a permis de concentrer les acides multiramifiés à structure isoprénique dans le filtrat final, où ils ont été aisément mis en évidence alors qu'ils sont indécelables par une analyse classique directe. Or la détection de ces acides peut apporter des informations utiles dans le domaine du contrôle. Les acides existent en effet dans les graisses d'origine marine, dans les graisses des ruminants, dans 
les lipides bactériens et, dans le cas du syndrome de Refsum, dans certains tissus humains. Dans la matière grasse du lait des mammifères les deux formes isomériques de l'acide pristanique et de l'acide phytanique sont en proportions équivalentes alors que la forme LDD prédomine dans les lipides d'origine marine et que seule la forme isomérique DDD est trouvée dans les bactéries [4]. Les acides isopréniques étant saturés, ne sont pas modifiés par un traitement d'hydrogénation et leur présence dans un mélange gras ou dans une margarine peut faciliter la reconnaissance d'huile de poisson. Dans I'étude présente, les proportions des formes isomères LDD et DDD sont équivalentes. Dans la matière grasse du lait de bufflonne les proportions respectives des acides 4 , 8, 12-triméthyltridécanoïque, pristanique et phytanique et indiqués dans le tableau 3 sont exactement similaires aux proportions de ces mêmes acides déterminés dans la matière grasse du lait de vache [22]. Dans la matière grasse du lait de bufflonne la proportion d'acide phytanique est environ dix fois plus élevée que la proportion de l'acide 4,8,12-triméthyltridécanoïque; ceci est également observé dans le cas de la matière grasse du lait de vache $[17,38]$.

\section{Les composés liposolubles}

Dans l'étude présente le cholestérol, le desmostérol, le lanostérol et le lathostérol ont été identifiés avec certitude dans la matière grasse du lait de bufflonne. Ces différents composés sont relativement proches puisque les deux derniers constituent des intermédiaires dans la synthèse du cholestérol à partir du squalène $[6,25]$.

$\mathrm{Au}$ cours de l'étude présente, le $\beta$-sitostérol n'a pas été identifié. Ce composé a cependant été signalé dans la matière grasse du lait de vache $[8,28,40]$ et également dans la matière grasse de lait de bufflonne [27]. De la même façon, ni le campestérol, ni le stigmastérol n'ont été décelés dans la matière grasse du lait de bufflonne au cours de l'étude rapportée ici alors que ces stérols ont été identifiés par Mincione et al. [27] antérieurement. Un certain nombre de remarques doivent être faites à ce sujet. La première remarque est d'ordre méthodologique. Mincione et al. [27] isolent les stérols après fractionnement par chromatographie sur couche mince de la fraction insaponifiable et effectuent ensuite l'analyse par chromatographie en phase gazeuse après triméthylsilylation. Or il a été constaté à diverses reprises [12, 23] que cette manière de procéder conduit à une très nette surestimation du taux des stérols, probablement "contaminés" par d'autres composés de même $\mathrm{R}_{\mathrm{f}}$. Par exemple les taux des stérols trouvés dans le lait de femme par Czeglédi-Janko [12], et repris par Morrison [32] et Jenness [20] sont près de 10 fois plus élevés que les taux antérieurement signalés par Macy et Kelly [26].

Il est par ailleurs assez surprenant que le taux de campestérol soit (comme sur le schéma présenté par Mincione et al. [27]) supé- 
rieur au taux de $\beta$-sitostérol. Cela ne semble en effet, n'avoir jamais été observé dans les produits naturels analysés jusqu'ici. Les auteurs japonais [40] indiquent une valeur moyenne (sur 264 échantillons de lait de vache) de 0,26 et 0,49 p. 100 des stérols totaux respectivement pour le campestérol et le $\beta$-sitostérol. Mincione et al. [27] n'indiquent pas par ailleurs l'identité du pic situé entre le cholestérol et le campestérol qui représente 2,73 des stérols totaux. Le $\beta$-sitostérol étant considéré comme un marqueur des huiles ou graisses végétales, il est important que soit établi avec précision le taux maximal de $\beta$-sitostérol naturellement présent dans la matière grasse du lait de vache, comme d'ailleurs dans la matière grasse du lait de bufflonne. Récemment, Homberg et Seher [18] affirment ne pas avoir trouvé de $\beta$-sitostérol dans la matière grasse de beurre. L'utilisation simultanée de deux méthodologies différentes appliquées au lait ou au beurre fabriqué avec ce lait (complexation des stérols avec la digitonine selon la norme FIL n. 32, ou isolement par chromatographie sur couche mince et dosage en présence d'un étalon interne selon Blanchard et al. [7]) permettrait d'aboutir à des résultats irrécusables et de lever certaines incertitudes actuelles. Bien d'autres composés liposolubles existent dans le lait de bufflonne comme ils existent dans le lait de vache et mériteraient d'être systématiquement étudiés: tels les alcools ou les hydrocarbures.

\section{Les acides gras monoramifiés autres que les acides iso et antéiso}

Ces acides ont été signalés par Ryhage [36] dans la matière grasse du lait de vache. Bien qu'identifiés alors par spectrométrie de masse ils n'avaient cependant pas été trouvés par Strocchi en 1971 [39] lors d'une étude consacrée aux acides monoramifiés de la matière grasse du lait de vache.

En 1972, Ackman et al. [5] entreprirent une nouvelle recherche de ces acides et parvinrent à les isoler par chromatographie en phase gazeuse (utilisation d'une phase stationnaire de succinate de butanediol et de 2 colonnes capillaires de $50 \mathrm{~m}$ environ montées en série) et à les identifier par spectrométrie de masse (acides comptant 15, 17 et 19 atomes de carbone). Dans la présente étude ils n'ont pas été identifiés.

\section{Ré s u m é}

L'étude a porté sur un échantillon de matière grasse obtenue à partir du lait de mélange produit par un troupeau italien de bufflonnes.

Les esters méthyliques ont été préparés par transestérification alcaline à partir de la matière grasse totale; les composés liposolubles autres que les esters méthyliques sont aussi présents. 
L'utilisation de la complexation à l'urée selon la méthode décrite par Iverson et al. [19] a permis de concentrer dans différentes fractions les esters méthyliques d'acides gras de structure similaire facilitant ainsi leur séparation (sur colonne capillaire d'Apiezon L) et leur identification par couplage chromatographie en phase gazeuse et spectrométrie de masse.

Le filtrat final du fractionnement à l'urée a été étudié après purification sur colonne de gel de silice.

On a ainsi isolé et identifié les acides gras isopréniques à 16 (acide 4, 8, 12-triméthyltridécanoïque), 19 (acide 2,6,10, 14-tétraméthylpentadécanoïque) et 20 atomes de carbone $(3,7,11,15$-tétraméthylhexadécanoïque) et les isomères LDD et DDD de ces deux derniers.

Les proportions relatives des différents acides gras ont été calculées. Elles s'alignent sur les proportions relatives connues pour le lait de vache. Cependant on a trouvé une proportion plus élevée de certains acides saturés à longue chaîne dans le lait de bufflonne que dans le lait de vache. En particulier le rapport 24:0/26:0 pourrait être utilisé comme critère de différenciation entre ces deux laits.

Parmi les composés liposolubles on a identifié par spectrométrie de masse, à côté du cholestérol, le lanostérol, le desmostérol et le lathostérol et une cétone de structure stéroïde. On n'a pas décelé la présence de $\beta$-sitostérol ou de campestérol.

\section{S u $\mathrm{m}$ m a ry}

CONTRIBUTION TO THE STUDY OF LIPOSOLUBLE COMPOUNDS

IN BUFFALO MILK

A sample of bulk milk fat from an italian water buffalo herd was studied.

Methyl esters were prepared by alkaline transesterification of total fat; besides methyl esters, minor liposoluble compounds were also present.

Using the urea-methyl esters complex formation according to the method described by Iverson et al. [19] it was possible to concentrate fatty acids methyl esters in different fractions thus facilitating their separation (on a glass capillary column coated with Apiezon L) and their identification by combined gas-liquid chromatography to mass spectrometry.

The final filtrate of the fractionation was studied after purification on a silica gel column.

Isoprenic fatty acids with 16 (4,8, 12-trimethyltridecanoic acid), $19(2,6,10,14$-tetramethylpentadecanoic acid) and 20 carbon atoms (3, 7, 11, 15-tetramethylhexadecanoic acid) and LDD and DDD isomers of the latter two, were thus isolated and identified. 
The relative proportions of the different fatty acids were calculated. They were similar to those found in cow's milk. However, a higher proportion of some saturated long-chain acids were observed in buffalo milk in comparison with cow's milk. The 24:0/26:0 ratio might thus be used as a differentiation criterion for milk from these two species.

Using mass spectrometry, we identified among the liposoluble components in addition to cholesterol, compounds as lanosterol, desmosterol and lathosterol as well a ketone with steroidal structure. The presence of $\beta$-sitosterol or campesterol was not detected.

Reçu pour publication en novembre 1980.

\section{Bibliographie}

[1] Abrahamsson (S.), Stallberg-Stenhagen (S.) and Stenhagen (E.) (1963). In progress in the chemistry of fats and other lipids. Vol. 7, Edit. R. T. Holman, Pergamon Press, Oxford and New York, p. 47.

[2] Albonico (F.), Addeo (F.), Alexandris (B.), Ameno (M.) e Mincione (B.) (1968). - Composizione dei latti di miscela prodotti in diverse condizioni e zone di allevamento delle bufale. L'Industria del Latte (Lodi), 4, 221226 (1967: 1, 10-12).

[3] Albonico (F.), Mincione (B.) e Casillo (R.) (1968). - Ricerche sul latte di bufala. III. Contributo alla conoscenza della composizione acidica del grasso. Prod. Anim., 7, 269-290.

[4] Ackman (R. G.) and Hansen (R. P.) (1967). - The occurrence of diastereomers of phytanic and pristanic acids and their determination by gasliquid chromatography. Lipids, 2, 357-362.

[5] Ackman (R. G.), Hooper (S. N) and Hansen (R. P.) (1972). - Some monomethyl-branched fatty acids from ruminant fats: open-tubular GLC separations and identifications of substitution on even-numbered carbon. Lipids, 7, 683-691.

[6] Attalah (A. M.) and Nicholas (H. J.) (1974). - Function of steryl esters in plants: a hypotesis that liquid crystalline properties of some steryl esters may be significant in plant sterol metabolism. Lipids, 7, 613-622.

[7] Blanchard (F.), Castang (J.), Derbesy (M.), Estrenne (J.), Olle (M.) et SolẼRE (M.) (1979). - Etude de la fraction stérolique des margarines végétales. Ann. Fals. Exp. Chim., n. 771, 25-37.

[8] Brewington (C. R.), Caress (E. A.) and Schwartz (D. P.) (1970). - Isolation and identification of new constituents in milk fat. J. Lipid Res., 11, 355-361.

[9] Brooks (C. J. W.), Harving (E. C.) and Young (J. S.) (1968). - Characterization of sterols by gas-chromatography - mass-spectrometry of the trimethyl silylethers. Lipids, 3, 391-402.

[10] Cerutti (G.), Finoli (C.), Vecchio (A.) e Mannino (S.) (1978). - Latti di bufala, di pecora e di capra: composizione e residui clororganici. Il Latte, 3, 310-318.

[11] Cucurachi (A.), Lotro (A.) (1968). - Indagine sul grasso di latte di bufala mediante gas cromatografia. Rivista Italiana delle Sostanze Grasse, 45, 171-174.

[12] Czegledi-Janko (von G.) (1965). - Mikropräparative Trennung der Lipidklassen von milch und blutserum. Z. Klin. Chem., 3, 14-20. 
[13] Eight peak index of mass spectra. Published by mass spectrometry. Data Centre, AWRE, Aldermaston, Reading (U.K.), 1974.

[14] El Rafey (M. S.) (1962) cited by Laxminarayana (H.), Dastur (N. N.) (1968), - Buffaloes'milk and milk products. Dairy Sci. Abstr., 30, 177-186 (Part I) ; 231-241 (Part II).

[15] EPA/NIH mass spectral data base. U.S. Department of Commerce National Bureau of Standards, S. R. Heller, J.W. A. Milne Ed. NSRDS-NBS, 63, dec. 1978, Washington.

[16] Gallacier (J. P.), Barbier (J. P.) et Kuzdzal-Savoie (S.) (1974). - Variations saisonnières des proportions relatives des acides gras d'un beurre de laiterie d'Ille-et-Vilaine. Le Lait, 54, 117-138.

[17] HanSEN (R. P.) (1964). - The isolation and identification of 4, 8, 12-trimethyl tridecanonic acid from butterfat. Nature, Lond. 201, 192.

[18] Homberg (E.) and SEHer (A.) (1980). - Sterol analysis for the detection of adulteration of milk fat. J.A.O.C.S., 57, Abstr. 146.

[19] Iverson (J. L.), Eisner (J.) and Firestone (D.) (1965). - Detection of trace fatty acids in fats and oils by urea fractionation and gas-liquid chromatography. J. Am. Oil Chem. Soc., 42, 1063-1068.

[20] Jenness (R.) (1974). - The composition of milk. In "Lactation. A comprehensive treatise ». B. L. Larson, V. R. Smith Ed., III, 3-107.

[21] Jensen (R. G.) (1973). - Composition of bovine milk lipids. J. Am. Oil Chem. Soc., 50, 186-192.

[22] Jensen (R. G.), Quinn (J. G.), Carpenter (D. L.) and SAmpugna (J.). (1967). Gas liquid chromatographic analysis of milk fatty acids: a review. J. Dairy Sci., 50, 119-126.

[23] Kuzdzal-Savore (S.) (1972). - Dosage de la matière grasse du lait. Revue Laitière Française, 677-687.

[24] Kuzdzal-Savoie (S.) et Raymond (J.) (1966). - Les acides gras " trans" du beurre. Isolement et dosage. Annls. Biol. Anim. Biochim. Biophys., 5, 497-511.

[25] Lederer (E.) (1970). - Cours de biochimie : Lipids. Ediscience, Paris, 50-61.

[26] MaCy (I. G.) and Kelly (H. J.) (1961). - Human milk and cows' milk in infant nutrition. In "Milk: the mammary gland and its secretion". S. K. Kon, A. T. Cowie ed., Academic Press, London, II, 265-304.

[27] Mincione (B.), Spagna Musso (S.), Stingo (C.), et Franciscis (G. de) (1976). Recherches sur le lait de bufflonne. 12. Les stérols. Milchwissenschaft, $31,282-287$.

[28] Mincione (B.), Spagna Musso (S.) et Franciscis (G. de) (1977). - Recherches sur les laits de différentes espèces. 2. Les teneurs stérols dans les laits de vache. Milchwissenschaft, 32, 599-603.

[29] Mincione (B.), Scudiero (A.), Casillo (R.) e Albonico (F.) (1972.) Ricerche sul latte di bufala. I fosfolipidi. Industria Agrarie, 10, 455-462.

[30] Moreno (M. M.) and Roncero (A.M.) (1964). - The application of urea inclusion coumponds in fat analysis. In "Analysis and characterization of oils, fats and fat products». M. A. Boeckenoogen ed., publi., London, 1, 95-118.

[31] MoRrison (W. R.) (1968). - The distribution of phospholipids in mammalian milks. Lipids, 3, 101-103.

[32] Morrison (W. R.) (1970). - Milk lipids in "Topics in lipid chemistry". F. D. Gunstone ed., Logos pr. Ltd., London, III, 51-106. 
[33] Morrison (W. R.), SMITH (L. M.) (1967). - Fatty and composition of milk phospholipids. II. sheep, indian buffalo and human milks. Lipids, 3, 178-182.

[34] Nota (G.), Goretti (G. C.), Armenante (M.) and Marino (G.) (1974). - An ultrasonic method for producing graphique - coated glass capillary column. J. Chromat., 95, 229-231.

[35] Ramamurthy (M. K.) and Narayanan (K. M.) (1972). - Polyinsaturated fatty acids of buffalo and cow milk fat. Milchwissenschaft, 27, 695-698.

[36] Ryhage (R.) (1967). - Identification of fatty acids from butterfat using a combined gas chromatograph-mass spectrometer. J. Dairy Res., 34, $115-121$.

[37] Ryhage (R.) and Stenhagen (E.) (1960). - Mass spectrometrie studies. Esters of monomethyl-substitued long chain carboxylic acids. Ark. Kemi., $15,291$.

[38] Sonneveld (W.), Haverkamp Begermann (P.), Beers (G. J. van), Keuning (R.) and Schogt (J.C.M.) (1962). - Tetramethylhexadecanoic and a constituent of butterfat. J. Lipid Res., 3, 351-355.

[39] Strocchi (A.) (1972). - Gli acidi grassi mono-metilramificati del grasso del burro. Scienza e Tecnologia degli Alimenti (S \& TA) (Bologna), 2, 55-59.

[40] Tsuchiya (F.), Yamamoto (Y.), Окabet (T.) and Hizawa (K.) (1972). Studies on cows' milk lipid. V. Sterols of milk lipid. J. Lipid Res., 43, 506-513 (DSA 36, 326, 1974). 